\title{
Sliding signal processing in telecommunication networks based on two-dimensional discrete Fourier transform
}

\author{
Vladimir Ponomarev ${ }^{1}$, Olga Ponomareva ${ }^{1, *}$, Alexey Ponomarev ${ }^{1}$, and Natalya Smirnova ${ }^{2}$ \\ ${ }^{1}$ Kalashnikov Izhevsk State Technical University, Studencheskay str., 7, Izhevsk, Udmurt Republic, \\ 426069, Russian Federation \\ ${ }^{2}$ Sevastopol State University, Universitetskaya str., 33, Sevastopol, 299053, Russian Federation
}

\begin{abstract}
A method of vertical sliding processing of two-dimensional discrete signals in the spatial frequency domain is proposed - a method of fast vertically sliding two-dimensional discrete Fourier transform. The mathematical representation of the two-dimensional discrete Fourier transform in algebraic and matrix form is considered. An effective method of vertically sliding two-dimensional discrete Fourier transform is proposed. The algorithm developed in the framework of the proposed method allows calculating the coefficients (bins) of this transformation in real time.
\end{abstract}

\section{Introduction}

It is difficult to overestimate the role and place of digital spectral processing of discrete one-dimensional (1-D) and two-dimensional (2-D) signals in telecommunication networks (telephone, computer, television networks and radio networks). The importance and relevance of the development and improvement of the methods of digital spectral processing of 1-D and 2-D signals is increasing due to the creation of telecommunication universal multiservice networks that can equally effectively transmit any type of information: data, sound and video.

The classical method of spatial-frequency processing of two-dimensional discrete signals is the 1-D and 2-D discrete Fourier transform (DFT), which allows to obtain a 1-D and 2-D frequency spectrum [1-13]. At the same time, there are a number of applications [9-12] where it is necessary to find the values of the frequency spectrum not at all 1-D and 2-D frequencies, but at a subset of them. In this case, the application of the full version of 1-D and 2-D DFT, even on the basis of the fast Fourier transform (FFT), becomes ineffective, since most of the obtained 1-D and 2-D DFT coefficients are not used. The solution to this problem for 1-D signals is given in [9].

The article discusses the solution of this problem for 2-D signals, introduces the concept of moving spatial-frequency processing based on 2-D DFT. Specifically, the vertical spatial-frequency processing of 2-D signals is considered.

*Corresponding author: ponva@mail.ru 


\section{Direct two-dimensional discrete Fourier transform}

Suppose we are given a discrete two-dimensional signal $x\left(n_{1}, n_{2}\right)$ in the form of a twodimensional sequence of finite length $0 \leq n_{1} \leq\left(N_{1}-1\right)$ and $0 \leq n_{2} \leq\left(N_{2}-1\right)$ or with a matrix of size $n_{1} \times n_{2}$ in a rectangular reference zone (specifically on a plane).

The direct two-dimensional discrete Fourier transform (2-D DFT) of a two-dimensional signal $x\left(n_{1}, n_{2}\right)$ is a special case of a direct two-dimensional z-transform:

$$
S_{N_{1}, N_{2}}\left(k_{1}, k_{2}\right)=\left.X\left(z_{1}, z_{2}\right)\right|_{z_{1}=W_{N_{1}}^{k_{1}}, z_{2}=W_{N_{2}}^{k_{2}}} ;
$$

and can be specified both in algebraic and in matrix form.

Algebraic form:

$$
\begin{gathered}
S_{N_{1}, N_{2}}\left(k_{1}, k_{2}\right)=\frac{1}{N_{1} \cdot N_{2}} \sum_{n_{1}=0}^{N_{1}-1} \sum_{n_{2}=0}^{N_{2}-1} x\left(n_{1}, n_{2}\right) \exp \left[-j 2 \pi\left(\frac{k_{1} n_{1}}{N_{1}}+\frac{k_{2} n_{2}}{N_{2}}\right)\right]= \\
=\frac{1}{N_{1} \cdot N_{2}} \sum_{n_{1}=0}^{N_{1}-1} \sum_{n_{2}=0}^{N_{2}-1} x\left(n_{1}, n_{2}\right) \cdot W_{N_{1}}^{k_{1} n_{1}} \cdot W_{N_{2}}^{k_{2} n_{2}}
\end{gathered}
$$

where $k_{1}=\overline{0,\left(N_{1}-1\right)}, k_{2}=\overline{0,\left(N_{2}-1\right)}$ are spatial frequencies; $x\left(n_{1}, n_{2}\right)$-two-dimensional signal; $n_{1}=\overline{0, N_{1}-1}, n_{2}=\overline{0, N_{2}-1}$;

$W_{N_{2}}^{k_{2} n_{2}}=\exp \left(-j \frac{2 \pi}{N_{2}}\left(k_{2} n_{2}\right)\right) ; S_{N_{1}, N_{2}}\left(k_{1}, k_{2}\right)-$ coefficients (bins) of 2-D DFT (twodimensional vector spatial-frequency spectrum).

\section{Matrix form:}

$$
S_{N_{1} \times N_{2}}=\frac{1}{N_{1} \cdot N_{2}} F_{N_{1} \times N_{1}}^{(2)} \cdot X_{N_{1} \times N_{2}} \cdot F_{N_{2} \times N_{2}}^{(1)}
$$

where

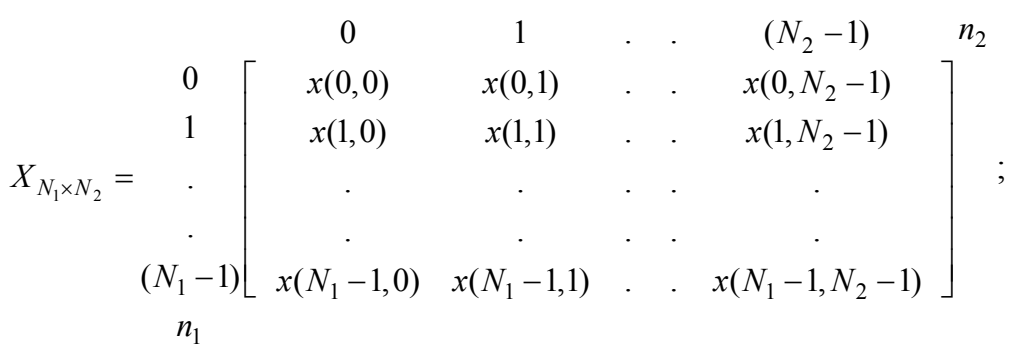

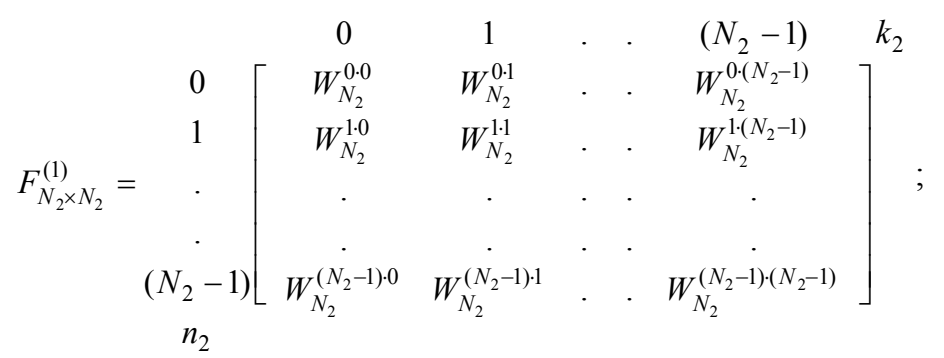




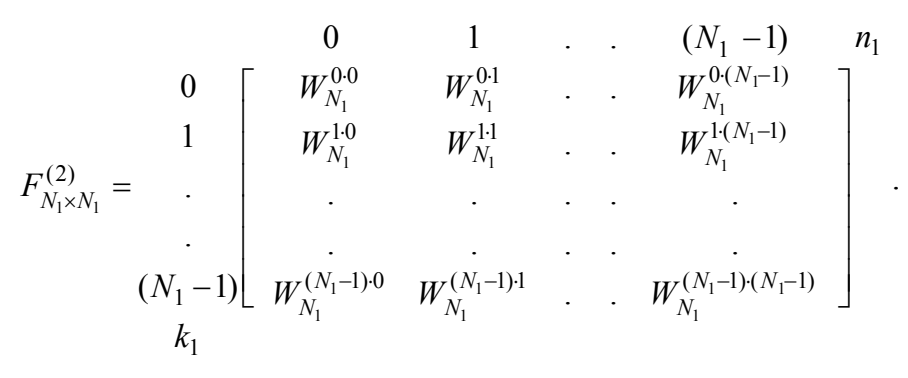

Hereinafter there is no loss of generality in omitting the multiplier $1 /\left(N_{1} \cdot N_{2}\right)$ in (3).

Due to the fact that for the product of the matrices (3) holds the associative property:

$$
S_{N_{1} \times N_{2}}=F_{N_{1} \times N_{1}}^{(2)} \cdot\left[X_{N_{1} \times N_{2}} \cdot F_{N_{2} \times N_{2}}^{(1)}\right]=\left[F_{N_{1} \times N_{1}}^{(2)} \cdot X_{N_{1} \times N_{2}}\right] \cdot F_{N_{2} \times N_{2}}^{(1)}
$$

and the 2-D DFT core is separable, then, according to (7), you can get the 2-D DFT bins $S_{N_{1} \times N_{2}}, k_{1}=\overline{0,\left(N_{1}-1\right)}, k_{2}=\overline{0,\left(N_{2}-1\right)}$, in two ways, each consists of two stages.

It is easy to find out that for obtaining $S_{N_{1}, N_{2}}\left(k_{1}, k_{2}\right) k_{1}=\overline{0,\left(N_{1}-1\right)}, k_{2}=\overline{0,\left(N_{2}-1\right)}$ it is necessary to perform $N_{1} \cdot N_{2}$ one-dimensional 2-D DFTs, for calculating which the algorithms of the fast Fourier transform - FFT can be effectively applied.

\section{Sliding spatial-frequency processing of discrete signals}

Let us consider the spatial-frequency processing of two-dimensional discrete signals in a sliding spatial analysis window. In contrast to the one-dimensional case for the twodimensional case, there are 4 possible ways of sliding of the spatial analysis window on the original two-dimensional discrete signal:

- $1^{\text {st }}$ way consists of horizontal right shift (HS +) and horizontal left shift (HS-);

- $2^{\text {nd }}$ way consists of vertical shift up (VS +) and vertical shift down (VS-);

- $3^{\text {rd }}$ way consists of right diagonal shift up (RDS + ) and right diagonal shift down (RDS-);

- $4^{\text {th }}$ way consists of left diagonal shift up (LDS + ) and left diagonal shift down (LDS-).

Fig. 1 shows a star diagram illustrating 4 types of sliding of a spatial analysis window using a discrete two-dimensional signal and examples of its shift.

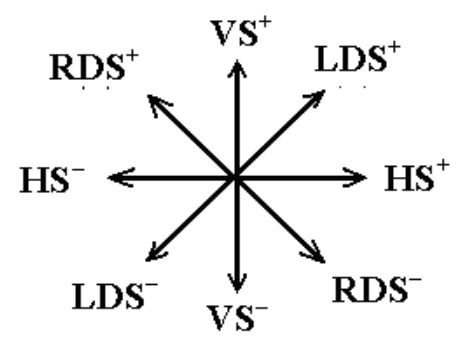

Fig. 1. The star chart illustrating 4 kinds of sliding of a spatial window. 
Suppose we need to find the coefficient (bin) of a two-dimensional discrete transform $S_{N_{1}, N_{2}}\left(k_{1} k_{2}\right)$ at the spatial frequency $\left(k_{1}, k_{2}\right)$ from the samples of the input signal $x\left(n_{1}, n_{2}\right)$. In this case, the matrix equation (3) is converted into the form:

$$
S\left(k_{1}, k_{2}\right)=\left[W_{N_{1}}^{k_{1} \cdot 0}, W_{N_{1}}^{k_{1} \cdot 1}, \ldots W_{N_{1}}^{k_{1} \cdot\left(N_{1}-1\right)}\right] \cdot\left\{X_{N_{1} \times N_{2}} \cdot\left[\begin{array}{c}
W_{N_{2}}^{0 \cdot k_{2}} \\
W_{N_{2}}^{1 \cdot k_{2}} \\
\cdot \\
\cdot \\
W_{N_{2}}^{\left(N_{2}-1\right) \cdot k_{2}}
\end{array}\right]\right\} .
$$

At the first stage, according to (8), we multiply the basis function of frequency $k_{2}$ and duration $N_{2}$ by a matrix of a discrete two-dimensional signal $x\left(n_{1}, n_{2}\right)$. As a result, we obtain a columned matrix $S_{N_{2}}\left(n_{1}, k_{2}\right)$ of size $N_{2}$, having spent on this procedure $N_{2} \cdot N_{1}$ complex multiplications and $\left(N_{2}-1\right) \cdot N_{1}$ complex additions. Further, at the second stage, we multiply the basis function of frequency $k_{1}$ and duration $N_{1}$ by a columned matrix of size obtained at the first stage, having spent on this procedure $N_{1}$ complex multiplications and $\left(N_{1}-1\right)$ complex additions.

Thus, it is necessary to expend $N_{2} \cdot\left(N_{1}+1\right)$ complex multiplications and $\left(N_{2}-1\right) \cdot\left(N_{1}+1\right)$ complex additions to obtain one coefficient of a two-dimensional discrete transform $S_{N_{1}, N_{2}}\left(k_{1} k_{2}\right)$ at spatial frequency $\left(k_{1}, k_{2}\right)$. Considering that performing one complex multiplication requires four real multiplications and two real additions, and one complex addition of two real additions, it is necessary to spend $4 \cdot N_{1} \cdot\left(N_{2}+1\right)$ real multiplications and $4 \cdot N_{1} N_{2}+2 \cdot\left(N_{1}-1\right)$ real additions to obtain the value of one coefficient of a two-dimensional discrete transformation $S_{N_{1}, N_{2}}\left(k_{1} k_{2}\right)$.

Note that this amount of computation needs to be performed at each shift of a twodimensional spatial analysis window using a two-dimensional signal. At the same time, it is easy to see that for any kind of shift of a two-dimensional signal a large number of values $X_{N_{1} \times N_{2}}$ of the complex matrix in the spatial analysis window remains unchanged.

Note that the shift of the spatial window along a two-dimensional discrete signal can be considered as a shift of a two-dimensional discrete signal in the spatial analysis window in the opposite direction to the movement of the spatial window.

\section{The algorithm of vertical sliding processing of 2-D signals based on 2-D DFT}

Let us need to find one coefficient (bin) of a two-dimensional discrete transformation $S_{N_{1}, N_{2}}\left(k_{1}, k_{2}\right)$ at a spatial frequency $\left(m_{1}, m_{2}\right)$ from the samples of the input signal $x\left(n_{1}, n_{2}\right)$. To obtain a bin, matrix equation (8) is converted to the form:

$$
S_{N_{1}, N_{2}}\left(m_{1}, m_{2}\right)=\left[W_{N_{1}}^{m_{1} \cdot 0}, W_{N_{1}}^{m_{1} \cdot 1}, \ldots W_{N_{1}}^{m_{1} \cdot\left(N_{1}-1\right)}\right] \cdot\left\{X_{N_{1} \times N_{2}} \cdot\left[W_{N_{2}}^{m_{2} \cdot 0}, W_{N_{21}}^{m_{2} \cdot 1}, . . W_{N_{2}}^{m_{2} \cdot\left(N_{1}-1\right)}\right]^{T}\right\} ;(9)
$$

where $T$ is the transpose symbol. 
The sequence of operations in the algorithm of vertical sliding processing of 2-D signals:

1. Find the column matrix $S_{N_{2}}\left(n_{1}, m_{2}\right)$ by size $N_{1}$, by multiplying the basis function duration $N_{2}$ and frequency $m_{2}$ on a matrix of a discrete two-dimensional signal $x\left(n_{1}, n_{2}\right)$.

2. Remember the column matrix $S_{N_{2}}\left(n_{1}, m_{2}\right)$ as a column matrix $S_{N_{2}}^{\left(n_{1}, m_{2}\right)}(0)$.

3. Calculate the 2-D coefficient of the discrete transform $S_{N_{1}, N_{2}}\left(m_{1}, m_{2}\right) \cdot$ by multiplying the column matrix $S_{N_{2}}\left(n_{1}, m_{2}\right)$ by a basis function of duration $N_{1}$ and frequency $m_{1}$. This stage ends the output of the algorithm to the operating mode.

4. Carry out the VS - shift of the discrete spatial window by one sample down the twodimensional signal $x\left(n_{1}, n_{2}\right)$ and obtain the matrix of the discrete two-dimensional signal $x\left[\left(n_{1}-1\right), n_{2}\right]$.

5. Form a column matrix according to the ratio;

$$
S_{N_{2}}^{\left(n_{1}, m_{2}\right)}(-1)=S_{N_{2}}^{\left(\left(n_{1}+1\right), m_{2}\right)}(0) ; n_{1}=\overline{0, N_{1}-2}
$$

6. Calculate the coefficient value of a two-dimensional discrete transformation $S_{N_{1}, N_{2}}\left(m_{1}, m_{2}\right)$ by multiplying the column matrix $S_{N_{2}}^{\left(n_{1}, m_{2}\right)}(-1)$ by a basis function of duration $N_{1}$ and frequency $m_{1}$.

7. Go to the implementation of paragraph No. 4.

\section{Conclusion}

The effectiveness of the proposed algorithm for vertical sliding processing of twodimensional discrete signals in the spatial frequency domain in comparison with the standard method for obtaining the coefficient of two-dimensional discrete transformation $S_{N_{1}, N_{2}}\left(m_{1}, m_{2}\right)$ :

- the standard algorithm (8) of the sliding processing of two-dimensional discrete signals in the space-frequency domain requires to obtain a coefficient $S_{N_{1}, N_{2}}\left(m_{1}, m_{2}\right)$ of twodimensional discrete conversion of $4 \cdot N_{1} \cdot\left(N_{2}+1\right)$ real multiplications $4 \cdot N_{1} \cdot N_{2}+2 \cdot\left(N_{1}-1\right)$ of real additions;

- the developed algorithm for the sliding processing of two-dimensional discrete signals in the space-frequency region after reaching the operating mode requires $4 \cdot\left(N_{1}+N_{2}\right)$ real multiplications and $4 \cdot\left(N_{1}+N_{2}-1\right)$ real additions to obtain a two-dimensional discrete transform coefficient $S_{N_{1}, N_{2}}\left(m_{1}, m_{2}\right)$.

\section{References}

1. W.K. Pratt, Digital image processing (4th edn. Wiley-Interscience publication, 2007)

2. S.L. Marpl, Digital Spectral Analysis with applications ( New Jersey, Prentice-hall, 1986)

3. L.P. Yaroslavsky, Compression, restoration, resampling, “compressive sensing”, J. Optics 17(7), 073001 (2015) 
4. M.N. Favorskaya, L.C. Jain, Development of mathematical theory in computer vision, (Springer International Publishing, Switzerland, 2015)

5. R.C. Gonzalez, R.E. Woods, Digital image processing (4th edn. Published by Pearson, 2018)

6. L. Rabiner, L., B. Gold, Theory and application of digital signal processing (New Jersey, Prentice-hall, 1975)

7. W. John, Multidimensional signal, image, and video processing and coding( Academic Press is imprint of Elsevier, 2006)

8. V.A. Ponomarev, O.V. Ponomareva, A.V. Ponomarev, Method for effective measurement of a sliding parametric fourier spectrum Optoelectronics, Instrumentation and Data Process. 50(2), p.p. 1-7 (2014)

9. O. Ponomareva, A. Ponomarev, V. Ponomarev, Evolution of forward and inverse discrete fourier transform, IEEE East-West Design \& Test Symp., pp. 313-318 (2018)

10. O. Ponomareva, A. Ponomarev, N. Ponomareva, Window - presume parametric discrete Fourier transform, IEEE East-West Design \& Test Symp., pp. 364-368 (2018)

11. O.V. Ponomareva, N. Ponomareva, Increased accuracy and extended functionality of digital filters based on frequency sampling, Devices and methodsof measuremants, v. 2(7), p.p. 114-119, (2013)

12. Favorskaya, M.N., Jain, L.C., Savchina, E.I.: Perceptually tuned watermarking using non-subsampled shearlet transform, Computer Vision in Control Systems-3: Aerial and Satellite Image Processing, ISRL, v. 135, pp. 41-69. (Springer International Publishing AG 2018)

13. Petrovsky, N.A., Rybenkov, E.V., Petrovsky, A.A.: Two-dimensional non-separable quaternionic paraunitary filter banks, IEEE Int. conf. on Signal Processing: Algorighms, Architectures, Arrangements, and Applications, Poznan, Poland, pp. 120125 (2018) 